\title{
Dynamic regulation of the cancer stem cell compartment by Cripto-1 in colorectal cancer
}

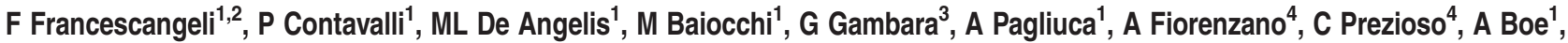 \\ M Todaro ${ }^{5}$, G Stassi ${ }^{5}$, NP Castro ${ }^{6}$, K Watanabe ${ }^{7}$, DS Salomon ${ }^{6}$, R De Maria ${ }^{2}$, G Minchiotti ${ }^{4}$ and A Zeuner ${ }^{*}, 1$
}

Stemness was recently depicted as a dynamic condition in normal and tumor cells. We found that the embryonic protein Cripto-1 (CR1) was expressed by normal stem cells at the bottom of colonic crypts and by cancer stem cells (CSCs) in colorectal tumor tissues. CR1-positive populations isolated from patient-derived tumor spheroids exhibited increased clonogenic capacity and expression of stem-cell-related genes. CR1 expression in tumor spheroids was variable over time, being subject to a complex regulation of the intracellular, surface and secreted protein, which was related to changes of the clonogenic capacity at the population level. CR1 silencing induced CSC growth arrest in vitro with a concomitant decrease of Src/Akt signaling, while in vivo it inhibited the growth of CSC-derived tumor xenografts and reduced CSC numbers. Importantly, CR1 silencing in established xenografts through an inducible expression system decreased CSC growth in both primary and metastatic tumors, indicating an essential role of CR1 in the regulation the CSC compartment. These results point to CR1 as a novel and dynamically regulated effector of stem cell functions in colorectal cancer.

Cell Death and Differentiation (2015) 22, 1700-1713; doi:10.1038/cdd.2015.19; published online 20 March 2015

Increasing evidence suggests that stemness is not a static condition, neither in normal cells nor in cancer. ${ }^{1,2}$ Spontaneous interconversion between states of higher and lower stemness has been observed both in embryonic stem cells (ESCs) and in adult tissues. ${ }^{3-6}$ In cancer, the transition between stem cells and non-stem cells is critical to the maintenance of a phenotypic equilibrium in which cell populations rapidly regulate relative hierarchic proportions in response to external stimuli. ${ }^{7}$ Stem cell dynamics have been particularly studied in the intestinal epithelium, where recent studies provided impressive insight on the behavior of normal stem cells. ${ }^{8}$ By contrast, the comprehension of stem cells dynamics in colorectal cancer (CRC) is at its beginning, although cancer stem cells (CSC) plasticity has been observed as the result of therapeutic and microenvironmental factors and proposed to influence patient outcome. ${ }^{9}$ In particular, the extracellular cues that regulate stem cell metastability in CRC remain largely unknown. Cripto-1 (CR1), also known as teratocarcinoma-derived growth factor-1 (TDGF-1), is an extracellular glycosylphosphatidylinositol (GPI)-anchored protein expressed in mouse and human ESCs, where it regulates stem cell differentiation. ${ }^{10}$ CR1 is usually low or absent in adult tissues but is reactivated in pathological conditions. Indeed, CR1 expression is rapidly induced in skeletal muscle upon acute injury and it is required in the muscle stem cell (satellite cell) compartment to promote efficient tissue regeneration. ${ }^{11} \mathrm{CR} 1$ is also overexpressed in several types of human tumors ${ }^{12}$ where it has a functional role in malignant transformation. ${ }^{13}$ Intriguingly, CR1 was found to be expressed in human ESCs with the highest self-renewal potential and was identified as a potential surface marker for an undifferentiated subpopulation in human embryonic carcinoma cells. ${ }^{14,15}$ We found that CR 1 is expressed by cells at the bottom of colonic crypts in normal human and mouse colon and by CSCs in human tumor tissues. In multicellular spheroid cultures of patient-derived colon cancer cells, CR1 expression was subject to a complex regulation at the intracellular, surface and secreted levels, which reflected the amount of selfrenewing cells. Furthermore, CR1 silencing decreased CSC numbers and tumor growth, pointing to a functional role of this protein in regulating the size of the CSC compartment.

\section{Results}

CR1 is expressed in stem cells compartments in normal colon and CRC. Colon cancer spheroids derived from primary human tumors have been previously demonstrated by our laboratory and others to be enriched in CSCs. ${ }^{16-18}$ Three CRC specimens (detailed in Supplementary Table S1) were obtained at the time of surgical resection and established as multicellular spheroid cultures in serum-free media. Spheroids were mainly composed by $\mathrm{CD}_{133^{+}}$cells, indicating that they are prevalently composed by stem

\footnotetext{
${ }^{1}$ Department of Hematology, Oncology and Molecular Medicine, Istituto Superiore di Sanità, Rome, Italy; ${ }^{2}$ Regina Elena National Cancer Institute, Rome, Italy; ${ }^{3}$ Department of Anatomy, Histology, Forensic Medicine and Orthopedics, Section of Histology and Medical Embryology, La Sapienza University, Rome, Italy; ${ }^{4}$ Institute of Genetics and Biophysics Adriano Buzzati-Traverso, CNR, Naples, Italy; ${ }^{5}$ Department of Surgical and Oncological Sciences, University of Palermo, Palermo, Italy; ${ }^{6}$ Mouse Cancer Genetics Program, National Cancer Institute, Frederick National Laboratory for Cancer Research, Frederick, MD, USA and ${ }^{7}$ Department of Medicine, University of California, Irvine, USA

*Corresponding author: A Zeuner, Department of Hematology, Oncology and Molecular Medicine, Istituto Superiore di Sanità, Viale Regina Elena, 299, Rome 00161, Italy. Tel: +39 06 49906060; Fax: +39 06 49387087; E-mail: a.zeuner@iss.it

Abbreviations: CR1, cripto-1; CSCs, cancer stem cells; ESCs, embryonic stem cells; TDGF-1, teratocarcinoma-derived growth factor-1; GPI, glycosylphosphatidylinositol; CRC, colorectal cancer; SDAC, spheroid-derived adherent cells; CK20, cytokeratin-20

Received 25.7.14; revised 27.1.15; accepted 02.2.15; Edited by A Zeuner; published online 20.3.15
} 
cells/transit-amplifying progenitors ${ }^{19}$ but also contained several cells positive for cytokeratin-20 (CK20) representing a more differentiated fraction. Culture in serum-containing medium led to cell adherence, loss of the AC133 epitope and widespread CK20 expression (Figure 1a and Supplementary Figure 1a). We analyzed the expression and localization of colon-specific and common stem cell markers in colon spheroids and spheroid-derived adherent cells (SDAC) and found that, among others, CR1 was strongly downregulated in SDAC both at the intracellular and at the surface level (Figure 1b, Supplementary Figure $1 \mathrm{~b}$ and Figure 1c, respectively). Flow cytometry analysis of CR1 expression showed variable levels of $\mathrm{CR}^{+}$cells in spheroid lines derived from different patients (between 8 and $33 \%$, Figure 1d). To investigate whether CR1 was associated with known stem cell markers in CRC spheroids, we analyzed its expression relative to Lgr5, Nanog and Ephrin B2 receptor (EphB2) and found that $\mathrm{CR} 1^{+}$cells represented a fraction of the EphB2 $2^{\text {high }}$ or $\mathrm{Nanog}^{+}$population and partly overlapped with Lgr5 $^{+}$cells (Figure 1e). To further investigate CR1 expression and localization in the colon, we analyzed tissue sections of normal and neoplastic colon and found that $\mathrm{CR} 1^{+}$ cells localized at the bottom of colonic crypts in normal mouse (both neonatal and adult, Figures if and g) and human colon (Figure 1h), whereas CR1 expression was deregulated in cancer, being widely present in neoplastic tissues of either mouse and human origin (Figures $1 \mathrm{~g}$ and $\mathrm{h}$ ). The expression of CR1 in the colon stem cell compartment was confirmed by double staining for CR1 and Lgr5 or EphB2. Double-positive CR1/Lgr5 ${ }^{+}$or $\mathrm{CR} 1 / \mathrm{EphB}^{\text {high }}$ cells were observed both in normal colon (in rare cells residing at the bottom of the crypts) and in colon adenocarcinoma, with a concentrated presence at the invasive front of the tumor suggesting a potential role for $\mathrm{CR}^{+}$cells in modulating epithelial-mesenchymal transition and metastatic spread (Figure 1h). The link between CR1 expression and colorectal CSCs was further supported by the observation that the expression of CR1 in CRC patients correlates with high expression of EphB2 or Lgr5 (Supplementary Figure 1c). These results confirm previous reports that CR1 is expressed in the neoplastic colon $^{12}$ and show for the first time its presence in the stem cell compartments of normal colon and CRC.

CR1 positivity in tumor spheroids oscillates in correspondence to increased stemness states. To gain further insights on the role of CR1 in CSCs, we monitored its expression in colon tumor spheroids over time and we found a marked oscillation of CR1 surface levels, which fluctuated between $\sim 1$ and $60 \%$ with a rough sinusoidal distribution (Figure $2 \mathrm{a}$ and Supplementary Figure 2a). Peaks of CR1 positivity corresponded to an increased clonogenic capacity of the whole spheroid population (Supplementary Figure 2a), suggesting a potential correlation between CR1 expression and stemness potential. In contrast, the expression of other CSC markers such as CD133 and Lgr5 remained constant over time (Supplementary Figure 2a). The fluctuation of CR1 expression was not influenced by oxygen conditions (Supplementary Figure $2 \mathrm{~b}$ ) and was not determined at the transcriptional level, as CR1 RNA did not show significant differences when monitored in parallel to surface expression (Supplementary Figure 2c). To explore the correlation between CR1 expression and stem cell properties, we separated spheroid cells positive and negative for CR1 expression at different time points and evaluated their ability to generate colonies in soft agar, assuming that cells with increased stemness capacity were those able to form larger colonies. We found a significant correlation between the generation of large colonies and the presence of CR1-positive cells (Figure 2b), indicating a causal correlation between CR1 positivity and clonogenic capacity. Then, we separated CR1-positive and CR1negative spheroid populations to monitor their behavior in liquid culture and to assess the presence of stem cellrelated features. Remarkably, purified $\mathrm{CR} 1^{+}$and $\mathrm{CR} 1^{-}$ populations showed rapid modifications of surface CR1 levels, with $\mathrm{CR}^{+}$cells undergoing an abrupt loss of CR1 expression (from 90 to $10 \%$ after 7 days) whereas $\mathrm{CR} 1^{-}$ cells acquired relatively high levels of CR1 expression (approximately from 0 to $40 \%$; Figure $2 \mathrm{c}$ ). After sorting, CR1 expression shortly recovered its tendency to oscillate, but with different phases in the two sorted populations. Finally, we assessed whether CR1-positive cells displayed enhanced stem cell features by analyzing Nanog and Lgr5 mRNA levels and $\beta$-catenin localization in sorted cells. We found that CR1-positive cells had increased levels of Nanog and Lgr5 and a significantly higher nuclear positivity of $\beta$-catenin (Figures $2 d$ and e), suggesting an enrichment of CSCs. Altogether, these observations suggest that CR1 levels are regulated in CSCs/progenitors both in basal conditions (in undisturbed spheroid cultures) and upon perturbation (after sorting), possibly reflecting fluctuations of the stem/progenitor cell compartment in response to intrinsic and extrinsic cues.

\section{Synchronized clonal fluctuations are responsible for CR1} oscillation in CSC-enriched cultures. To examine more in depth the dynamics of CR1 oscillation, we first monitored surface CR1 expression in three patient-derived spheroid lines, which all showed a rough sinusoidal trend although with different phases (Figure 3a). Then, we ought to understand whether the levels of CR1 were regulated at the clonal level and whether different clones in spheroid cultures displayed independent levels of CR1. To do this, we transduced cells derived from one spheroid line with vectors encoding for six different fluorescent proteins to allow multicolor clonal cell marking. ${ }^{20}$ Transduced cells were sorted and cloned, and single clones were subsequently assessed for the respective expression of fluorescent proteins, which displayed a remarkable stability over time as previously reported (Figure 3b). Colored clonal cell populations were then pooled (two pools of three clones each) and CR1 expression was monitored over time for each clone. We found that clones belonging to the same pooled population showed a similar trend of CR1 surface levels (Figure 3c), suggesting that the dynamics of CR1 expression are dictated by population forces rather than by independent clonal features.

Fluctuations of CR1 expression reflect a complex spatiotemporal distribution of the CR1 protein. To gain 
further insights into the process of CR1 fluctuation, we analyzed the presence of CR1 in different cellular compartments (cytoplasm, total cell membranes and plasmamembrane) and in the extracellular milieu, where it can be released to exert a paracrine activity on surrounding cells. $^{21,22}$ To do this, we collected lysates of subcellular fractions from cultured spheroid cells once a week for 5 weeks. We verified the purity of cellular fractions by immunoblotting against compartimentalized proteins and detected the presence of CR1 in the cytosolic and total membrane fractions, as expected (Supplementary Figure 3). In parallel, we monitored CR1 surface expression by flow cytometry and we assessed the levels of CR1 released in the extracellular medium by ELISA. Interestingly, we found that CR1 levels were subject to fluctuations not only on the cell surface but also in the cytoplasmic and total membrane compartments and in the extracellular medium (Figure 4). Higher levels of the unmodified form of CR1 in the cytoplasm corresponded to increased CR1 release and to a lower surface expression, while the modified (i.e., glycosylated and fucosylated ${ }^{23}$ ) protein was increased in correspondence to low extracellular levels and high-surface expression. These observations indicate that CR1 protein is subject to multiple levels of regulation, not only by posttranslational modifications but also by intracellular compartimentalization and extracellular release.

CR1 and the downstream activation of the Glypican-SrcAkt pathway are essential for CSC growth. To investigate the functional role of CR1 in colorectal stem-progenitor cells, we treated spheroid cell lines with small interfering RNAs (siRNAs) to induce a transient decrease in CR1 expression. CR1 siRNA were effective in reducing CR1 RNA levels and resulted in a significant inhibition of colony size in semisolid culture (Figures $5 a$ and $b$ ). Then, we carried out stable CR1 silencing by using lentiviral vectors containing two different shRNA sequences (detailed in Supplementary Table 2). The two sequences were both able to induce a strong reduction in CR1 RNA levels, although at a different extent, in spheroids (Pt1) as well as in NTERA2 teratocarcinoma cells (Figure $5 \mathrm{c}$ ). Functionally, the two shRNAs induced, respectively, a complete abrogation or a strong reduction of colony formation in soft agar (Figure 5d) and an equivalent growth inhibitory effect in liquid culture (Figure $5 \mathrm{e}$ ). Cell cycle analysis of CSC stably expressing CR1 shRNA4890 4 days after transduction showed an accumulation of cells in the G2/M phase of the cycle (from 11.6 to $26.2 \%$ ) and a corresponding decrease of cells in G1 (from 66.5 to $45.4 \%$; Figure $5 f$ ). The number of hypodiploid cells did not change in CR1-silenced CSC, but we observed a small increase in the percentage of debris/ sub-hypodiploid cells generally indicative of cell death (Debri/ Apo from 2.2 to $6.6 \%$; Figure 5f, right panels). CR1 has been demonstrated to signal through a Nodal-dependent and a Nodal-independent pathway, ${ }^{24}$ the latter being activated by CR1 binding to Glypican-1 and downstream activation of c-Src and Akt signaling. To gain insights into the pathway active downstream of CR1 in colon CSCs, we assessed the expression of ALK4, Nodal, Glypican-1 and the co-receptor GRP78 in spheroid cells and found that such factors were all expressed at the RNA and protein level (Supplementary Figure 4). Then, we evaluated the effect of CR1 silencing on the levels of CR1 signaling partners and of selected proteins involved in cell cycle and apoptosis regulation. Levels of CR1, phospho-Smad2, Glypican-1, phospho-Src and phospho-Akt sensibly decreased in silenced cells, whereas levels of Alk4 slightly increased. In CR1-silenced cells we also observed an increase in polo-like kinase 1 and cyclin D1, confirming the accumulation of cells in the G2/M phase of the cell cycle. In addition, we observed a small amount of cleaved PARP in silenced cells, indicating the occurrence of low levels of cell death (Figure $5 \mathrm{~g}$ ). These results suggest that CR1 is linked to the activation of the Glypican-Src-Akt pathway in colorectal CSCs/progenitors, although we cannot rule out that Nodaldependent signaling may have a role in other cellular systems and in patient tumors.

CR1 downregulation inhibits tumor progression and decreases CSC content in vivo. To investigate the effects of CR1 silencing in vivo, we employed a Tet-inducible lentiviral silencing system that allowed us to achieve CR1 silencing at different stages of tumor formation and progression. The sh4890 CR1 silencing sequence was cloned into the TRIPZ Tet-inducible lentiviral vector, which achieved $\sim 75 \%$ reduction of CR1 RNA levels as compared with controls (Figure 6a). CR1 downregulation inhibited spheroid proliferation and strongly reduced colony formation in semisolid culture (Figures $6 \mathrm{~b}$ and $\mathrm{c}$ ). Cells were then injected subcutaneously in three groups of immunecompromised (NOD/SCID $\gamma$-chain deficient, NSG) mice in order to compare the growth of tumors generated from control cells (transduced with the silencing construct but not induced with doxycycline, thereafter referred to as non-induced), from cells in which CR1 expression was silenced either at the time of injection (shCR1 induced from day 0 ) or at the appearance of palpable tumors (shCR1 induced from day 21). Tumor xenografts were then monitored for 1 month. Tumors generated by cells with CR1 silenced from day 0 grew at a significantly reduced growth rate (Figure 6d). Notably, also tumors in which CR1 expression was silenced at day 21 also had a significantly, although less pronounced, reduced growth rate compared

\footnotetext{
Figure 1 Cripto-1 is expressed in normal and neoplastic colon stem cells. (a) Immunofluorescence staining for CD133 and cytokeratin-20 (CK20) on colon cancer spheroids (stem) and sphere-derived adherent cells (SDAC). Line Pt1, $60 \times$ magnification, 1,4 $\times$ zoom (b) Immunofluorescence detection of stem cell antigens on permeabilized spheroids (Stem) and SDAC, line Pt2. $60 \times$ magnification, 2x zoom (c) Immunofluorescence detection of CR1 on non-permeabilized spheroids (Stem) and sphere-derived adherent cells (SDAC), line Pt2. $60 \times$ magnification (d) Flow cytometry analysis of CR1 expression on spheroid lines derived from different patients (detailed in Supplementary Table 1) (e) Flow cytometry staining of CR1 and either Nanog, Ephrin B2 receptor (EphB2) or Lgr5 on spheroid line Pt1. Numbers refer to the percentage of double-positive cells (f) Whole-mount in situ hybridization of CR1 on mouse neonatal colon. Magnification 20x, inset $40 \times(\mathrm{g})$ Anti-CR1 immunohistochemistry on adult mouse normal colon (left panel) and high-grade adenoma (right panel). Arrows indicate cells with membrane CR1 staining (h) Immunofluorescence staining of CR1/Lgr5 (left) and CR1/EphB2 (right) expression on FFPE sections of normal colon and colon cancer. Images of colon cancer were taken both from central areas (Colon cancer) and from the invasive edge of the tumor (Invasion front). Magnification $40 \times$, detail (yellow square) $3 \times$ zoom
} 

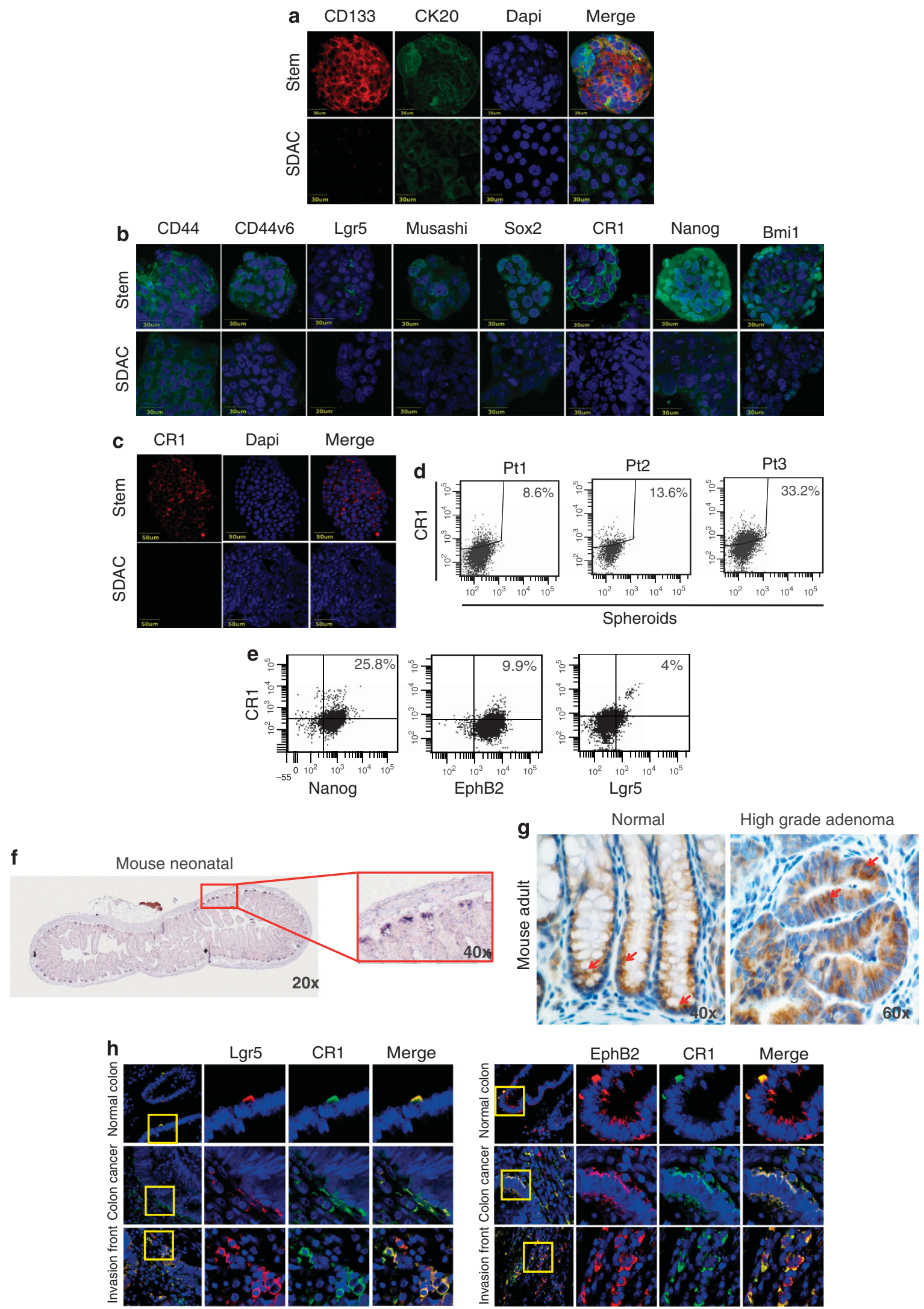

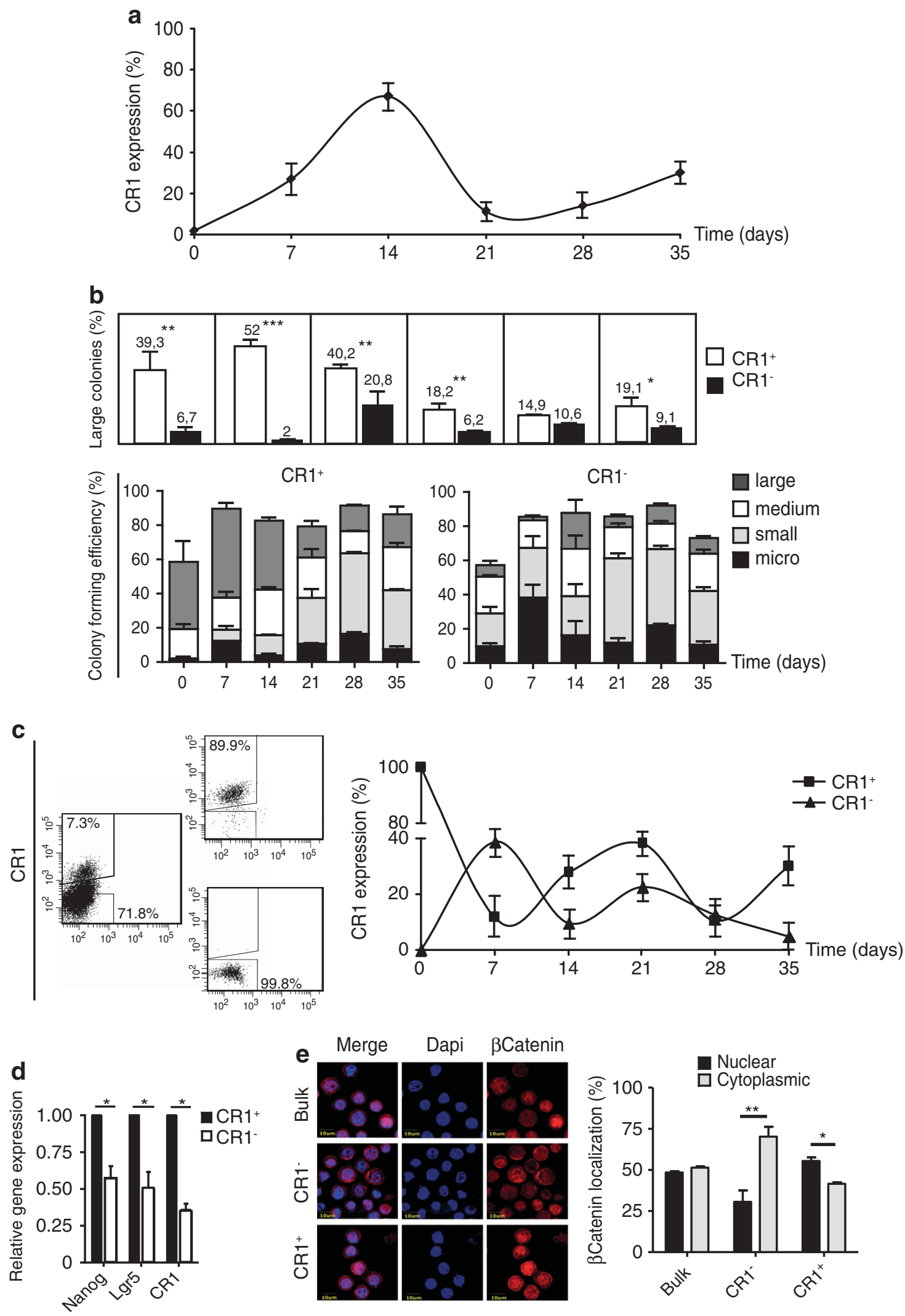
with controls (Figure 6d). Importantly, an ex vivo analysis of the clonogenic capacity of cells extracted from the xenografts showed that CR1 silencing strongly reduced the number of colony-forming cells (Figure 6e). Moreover, when we analyzed the effect of CR1 silencing with serial transplantation/ limiting dilution assays, we found that CR1-silenced cells gave rise to secondary tumors with increased latency, reduced volume and decreased frequency as compared with controls, indicating that CR1 downregulation affects the number of tumor-initiating cells (TICs) in vivo (Figure 6f). Stem cell quantification of results shown in Figure $6 f$ with the Extreme Limiting Dilution Analysis (ELDA) method ${ }^{25}$ indicated an estimated proportion of $1 / 18$ TICs in the Vector group and $1 / 69$ TICs in the CR1-silenced group $\left({ }^{\star} P<0.05\right)$. Altogether, these results suggest that $\mathrm{CR} 1$ has a role in both tumor initiation and progression through regulation of the CSC compartment.

CR1 is essential for CSC growth at metastatic sites. A subpopulation of colon CSC, which has been recently shown to be characterized by CD44v6 expression, possesses increased migratory and metastatic properties. ${ }^{26}$ Double staining of CSC spheroids for CR1 and CD44v6 showed that the majority of $\mathrm{CR}^{+}$cells expressed also CD44v6 (11.4\% $\mathrm{CR} 1^{+} \mathrm{CD} 44 \mathrm{v} 6^{+}$versus $3.9 \% \mathrm{CR} 1^{+}$ $\mathrm{CD}_{446^{-}}$; Figure $7 \mathrm{a}$ ). In line with a potential role of CR1 in the regulation of CSC migratory abilities, spheroid cells with silenced CR1 expression showed a significantly reduced migration in transwell assays (Figure 7b). To verify whether CR1 silencing would also affect metastatic growth in vivo, colon spheroids were double transduced with the inducible CR1-silencing vector and with a luciferase reporter vector to allow for tumor bioimaging. Transduced spheroid cells were then treated with doxycycline to induce CR1 silencing and injected in the tail vein of NSG mice, which received doxycycline in the drinking water to maintain CR1 silencing throughout the experiment. Four months after injection, noninduced control cells gave rise to lung metastases in all injected mice, whereas CR1-silenced cells produced very few metastases (Figure 7c), indicating an essential role of CR1 for CSC growth at metastatic sites. To provide additional support to a role of CR1 in metastatic CSC growth, we injected double-transduced spheroid cells in the spleen of NSG mice and we waited $\sim 1$ month to allow tumor formation (Figure 7d). Tumor-bearing mice were then splenectomized and divided into two groups, which were, respectively, left non-induced or administered doxycycline to activate CR1 silencing (Figure 7d). Mice were regularly monitored through bioimaging for the appearance and growth of liver metastasis. Control (non-induced) mice had a significantly increased rate of metastasis growth as compared with mice in which CR1 was silenced (Figure 7e), further supporting a role for CR1 in the growth of metastatic CSCs.

\section{Discussion}

In line with the emerging concept of stem cell plasticity in normal and neoplastic tissues, we identified the ESC marker CR1 as a protein dynamically expressed on CRC cells. The dynamic behavior of CR1 was apparent in multicellular spheroids of patient-derived cells (composed by a large majority of CD133 ${ }^{+}$ stem/progenitor cells) both in basal culture conditions and upon separation of CR1-positive and -negative cell populations, which rapidly changed CR1 surface expression. CR1 expressed on the membrane of colorectal stem/progenitor cells was not simply unstable but followed a roughly sinusoidal trend, reminescent of fluctuations in stemness regulators that have been shown to occur in ESCs and to have a key role in the maintenance of pluripotency. ${ }^{3,27-30}$ The parallel between the fluctuations of CR1 and that of stemness factors is reinforced by the observation that cells with the highest expression of CR1 have an increased clonogenic potential, indicating their enrichment in self-renewing elements. Notably, the fluctuation of cellular factors is generally attributed to oscillations of gene expression. By contrast, fluctuations of CR1 occurred upon small variations in transcript levels but in the presence of consistent changes in the subcellular and extracellular distribution of the protein. In this regard, we show for the first time that CR1 is released by CSCs/ progenitor cells, possibly representing a mechanism that contributes to a cross-talk between tumor cells. In line with this hypothesis, stemness has been recently described as a statistical property of cell populations (rather than a property of individual cells) that depends both from cell-intrinsic mechanisms and from cell-to-cell signals that act as system-level feedback mechanisms. ${ }^{31,32}$ Identifying the molecular cues of intercellular coordination will be essential to interfere with the adaptive responses of cancer cells to external challenges and ultimately to achieve long lasting therapeutic effects.

We showed that the cyclic fluctuation of surface CR1 reflects the synchronized expression pattern of different cell clones present in the same culture. This observation suggests the existence of feedback mechanisms that coordinate CR1 expression of clones coexisting in the same cell population. This phenomenon would result in the waves of high CR1 expression in the whole cancer cell population, corresponding to the waves of high clonogenic potential. Furthermore, this observation suggests the existence of a coordinated behavior in the cancer cell population, similar to the mechanisms of quorum sensing that have been previously postulated on the basis of mathematical models ${ }^{33}$ and experimentally observed in human cell lines. ${ }^{34}$

Figure 2 Cripto-1 expression is dynamically regulated on the surface of CSC/progenitor cells and is associated with stemness-related features. (a) Flow cytometry analysis of surface CR1 (indicated as the percentage of positive cells) in the colorectal spheroid line Pt1 at the indicated times of culture. Values are the mean \pm S.D. of technical triplicates (b) Self-renewal capacity of sorted Cripto-positive $\left(\mathrm{CR} 1^{+}\right)$and Cripto-negative $\left(\mathrm{CR} 1^{-}\right)$cells from spheroid line Pt1 at the indicated times of culture, evaluated as colony formation in semisolid culture and expressed in bottom panels as normalized colony size/percentage over plated cells. Top panel is a representative illustration of the percentage of large colonies only. ${ }^{*} P<0.05$; ${ }^{* *} P<0.01$, ${ }^{* *} P<0.001$ (c) FACS-based separation of $\mathrm{CR} 1{ }^{+}$and $\mathrm{CR} 1{ }^{-}$subpopulations of spheroid line Pt1 (left panel), CR1 surface expression immediately after sorting (central panels) and monitored for 5 weeks after sorting (right graph). Values are the mean \pm S.D. of technical triplicates (d) mRNA expression of CR1, Lgr5 and Nanog on sorted $\mathrm{CR} 1^{+}$and CR $1^{-}$populations. ${ }^{*} P<0.05$ (e) Subcellular localization of $\beta$-catenin on sorted $\mathrm{CR} 1^{+}$and $\mathrm{CR} 1^{-}$populations or mock-sorted (Bulk) cells from line Pt1 (left) and relative quantification of nuclear versus cytoplasmic $\beta$-catenin in the three samples (right). $60 \times$ magnification, $3,5 \times$ zoom. ${ }^{*} P<0.05 ;{ }^{* *} P<0.01$ 

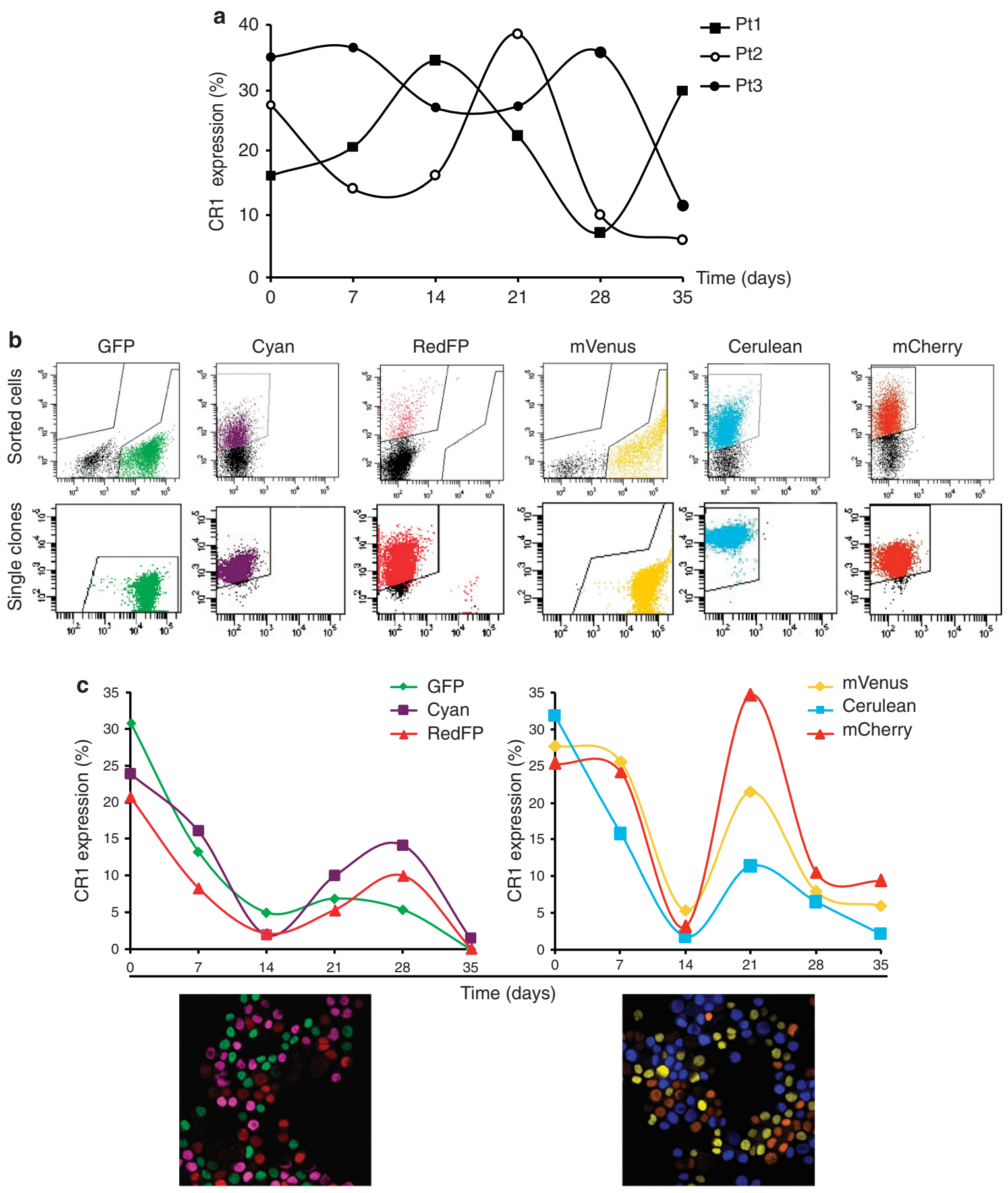

Time (days)

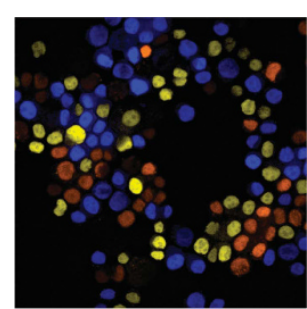

Figure 3 Cripto-1 fluctuation derives from the synchronized oscillation of CR1 expression in different cell clones. (a) Flow cytometry analysis of surface CR1 (indicated as the percentage of positive cells) at the indicated times of culture in three colorectal spheroid lines derived from different colorectal cancer patients (Pt1-Pt3) (b) Spheroid cells from line Pt1 were transduced with vectors coding for six different fluorescent reporters, sorted on the basis of fluorescent protein expression (upper panels), single-cell cloned and retested for reporter expression (lower panels) (c) Cell clones were pooled (two pools of three clones each) and monitored for 5 weeks for CR1 expression, indicated as the percentage of positive cells. Bottom panels: representative pictures of the pooled clones

In normal human and mouse colon, CR1 was expressed by cells located at the bottom of the crypts possibly representing a marker of normal colorectal stem cells. However, the role of CR1 in normal colon is still unknown, as CR1 knockout embryos die at an early stage due to gross defects in embryonic symmetry. ${ }^{35}$ The expression of CR1 was deregulated in CRC, as previously reported, ${ }^{36}$ being present on cancer cells particularly at the invasive front of the tumor. Despite its diffuse expression on cancer cells, our results show that the highest levels of CR1 expression are present in $\mathrm{Lgr}^{+}$cells and EphB2 ${ }^{\text {high }}$ cells, indicating that CR1 may have a specific role in CSC regulation. The supposed role of CR1 in CSCs seems corroborated by the observation that CR1 silencing profoundly affects signaling pathways essential for CSC growth. 

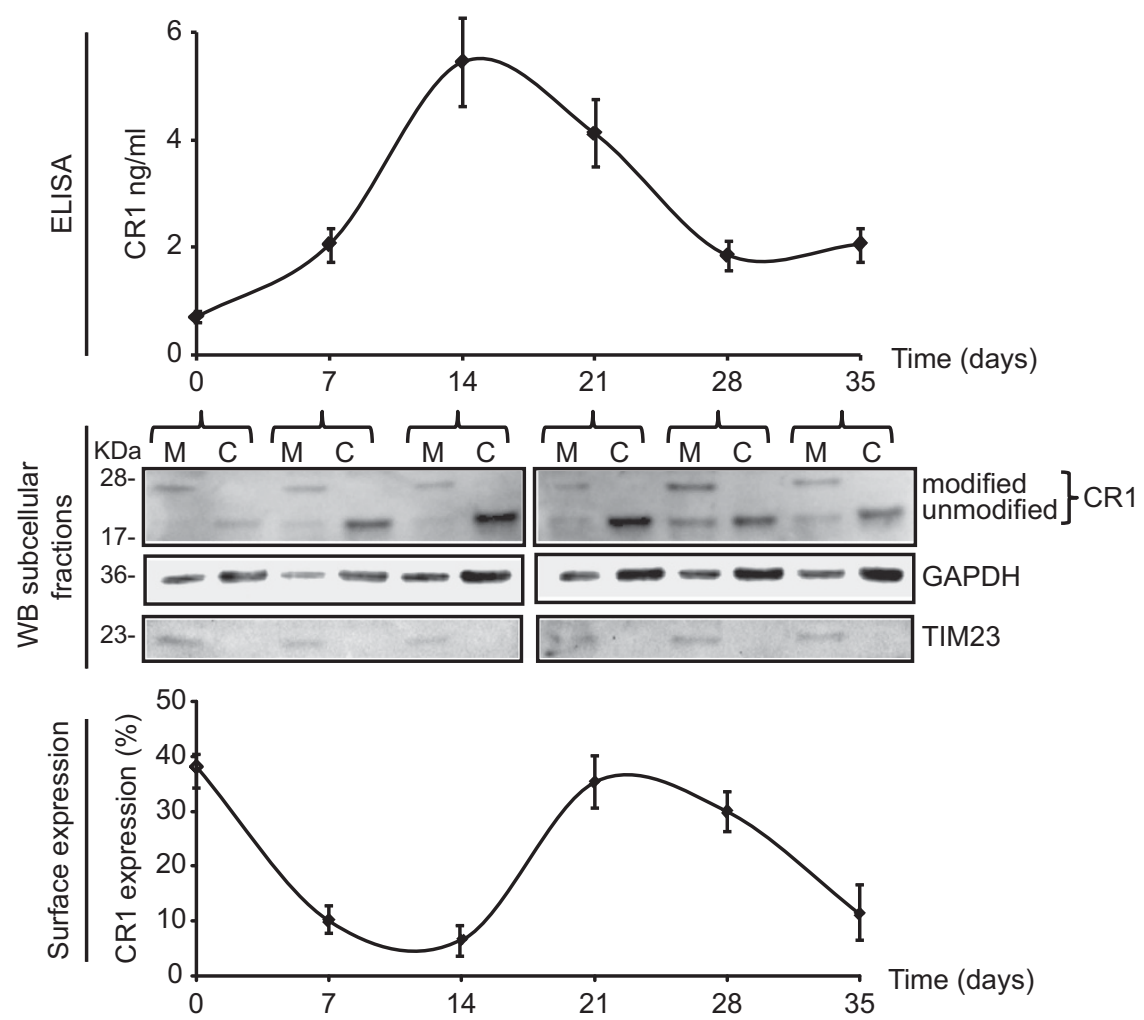

Figure 4 Cripto-1 fluctuation involves subcellular compartmentalization and extracellular release. Results shown derive from one representative experiment in which the spheroid line Pt1 was simultaneously monitored over time for levels of CR1 released in the extracellular medium as detected by ELISA (enzyme-linked immunosorbent assay; upper panel; values are the mean \pm S.D. of triplicate medium sampling), $C R 1$ presence in subcellular fractions of cytoplasm $(C)$ or total membranes (M; central panels) and CR1 surface expression detected by flow cytometry (lower panel, values are the mean \pm S.D. of technical triplicates). Higher and lower forms of CR1 (referred to as modified and unmodified) represent the posttranslationally glycosylated/fucosylated form of the protein and the non-modified form, respectively. GAPDH, glyceraldehyde 3-phosphate dehydrogenase; TIM23, translocase of the inner membrane

Specifically, CR1 silencing results in a strong decrease of pAkt, which has been recently indicated as a key factor in colorectal CSC survival specifically expressed by the tumorigenic/ metastatic fraction. ${ }^{26}$ Accordingly, our results show that a reduction of $\mathrm{CR} 1$ expression in tumor xenografts inhibits tumor growth and induces a decrease of CSC numbers. Moreover, we observed that CR1 silencing inhibited the growth of metastatic CSCs in mouse lungs and liver, suggesting that CR1 may be important for CSC expansion at metastatic sites. These findings may explain the correlation between elevated CR1 levels and the presence of metachronous metastases found in colon cancer patients, ${ }^{37}$ also corroborating the hypothesis that the state of the CSC compartment may be related to patient prognosis. ${ }^{38}$ Altogether, these results indicate CR1 as a new functional player of CRC stem/progenitor cell regulation and highlight the dynamic behavior of these cells, which should be taken into account in order to devise effective anticancer strategies.

\section{Materials and Methods}

Cells and tissues. Colon cancer specimens were obtained from patients undergoing surgical resection upon informed consent and approval by the Istituto Superiore di Sanità (ISS) Ethical Committee. Spheroid cultures were established in serum-free medium containing $20 \mathrm{ng} / \mathrm{ml}$ EGF and $10 \mathrm{ng} / \mathrm{ml}$ bFGF (PeproTech, Rocky Hill, NJ, USA) and subsequently kept on ultra-low attachment dishes (Nunc, Sigma-Aldrich, St. Louis, MO, USA) in a $5 \% \mathrm{CO}_{2}$ humidified incubator at $37^{\circ} \mathrm{C}$ as described. ${ }^{39}$ Spheroids were routinely tested for their ability to produce colon adenocarcinomas histologically identical to the human tumors of origin when injected

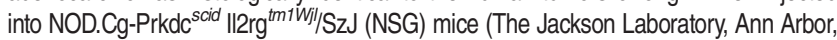
MI, USA). SDAC were obtained by culturing spheroid cells in $10 \%$ serum-containing DMEM (Gibco-Invitrogen, Carlsbad, CA, USA) for 10 days. Mouse colon samples were obtained either from healthy neonatal or adult animals or mice with sporadic high-grade adenoma (Apcflox; LSL-KrasG12D). Human colon samples were obtained from formalin-fixed paraffin-embedded (FFPE) surgical specimens of neoplastic and peritumoral (referred to as normal) tissue.

Antibodies and reagents. Polyclonal antibodies against CR1 used for immunofluorescence and flow cytometry analyses were produced by immunizing rabbits with purified recombinant soluble CR1 produced in 293 cells. ${ }^{40}$ Monoclonal antibodies against Lgr5 (both conjugated and unconjugated) and CD133 (AC133 epitope) were obtained from Miltenyi Biotec (Bergisch Gladbach, Germany). Antibodies against CD44v6, Nanog, Musashi, Sox2 and EphB2 (the latter both Alexa Fluor 488-conjugated and unconjugated) were obtained from R\&D System Inc. (Minneapolis, MN, USA). Anti-CK20 was from Dako (Carpinteria, CA, USA). Alexa Fluor-conjugated secondary antibodies were obtained from Invitrogen Molecular Probes. Anti-Alk4, cyclin D1, Glypican-1 and TIM23 were from Santa Cruz Biotechnology (Santa Cruz, CA, USA). Monoclonal anti- $\beta$-actin, GAPDH and PARP were from Sigma-Aldrich. Total Src, p-Src, p-AKT, total AKT, p-smad2, smad2, CD44, polo-like kinase 1, Bmi1 and $\beta$-catenin were from Cell Signaling Technology (Danvers, MA, USA). Secondary anti-mouse and anti-rabbit antibodies coupled to horseradish peroxidase were from GE Healthcare (Uppsala, Sweden).

Cell proliferation and self renewal assays. Cell proliferation was analyzed with the Cell Titer GLO assay (Promega, Madison, WI, USA) as described ${ }^{39}$ with a DTX880 plate reader (Beckman Coulter, Brea, CA, USA). For clonogenic assays, dissociated spheroids were plated in triplicate at $500 \mathrm{cells} /$ well suspended in $0.3 \%$ agarose over a layer of $0.4 \%$ agarose. Plates were incubated in a $5 \% \mathrm{CO}_{2}$ humidified incubator at $37^{\circ} \mathrm{C}$ and colony counts were performed 
20-25 days after plating. Colonies were stained with crystal violet $(0.1 \%$ in $10 \%$ $\mathrm{MetOH}$ ) and classified as follows: micro $<30 \mu \mathrm{m}$, small $30-60 \mu \mathrm{m}$, medium $60-90 \mu \mathrm{m}$ and large $>90 \mu \mathrm{m}$. Data represent the percentage of colony numbers relative to plated cells.
Constructs. For transient CR1 silencing, $1.25 \times 10^{5}$ spheroid cells were plated on 6-well plates in antibiotic-free culture medium and incubated for $4 \mathrm{~h}$ at $37^{\circ} \mathrm{C}$ in the presence of $320 \mathrm{nM}$ ON-TARGET PLUS TDGF-1 siRNA (Dharmacon, Lafayette, CO, USA; L-004832-00-0005) or control siRNA (Non-targeting siRNA D-001810-01), both
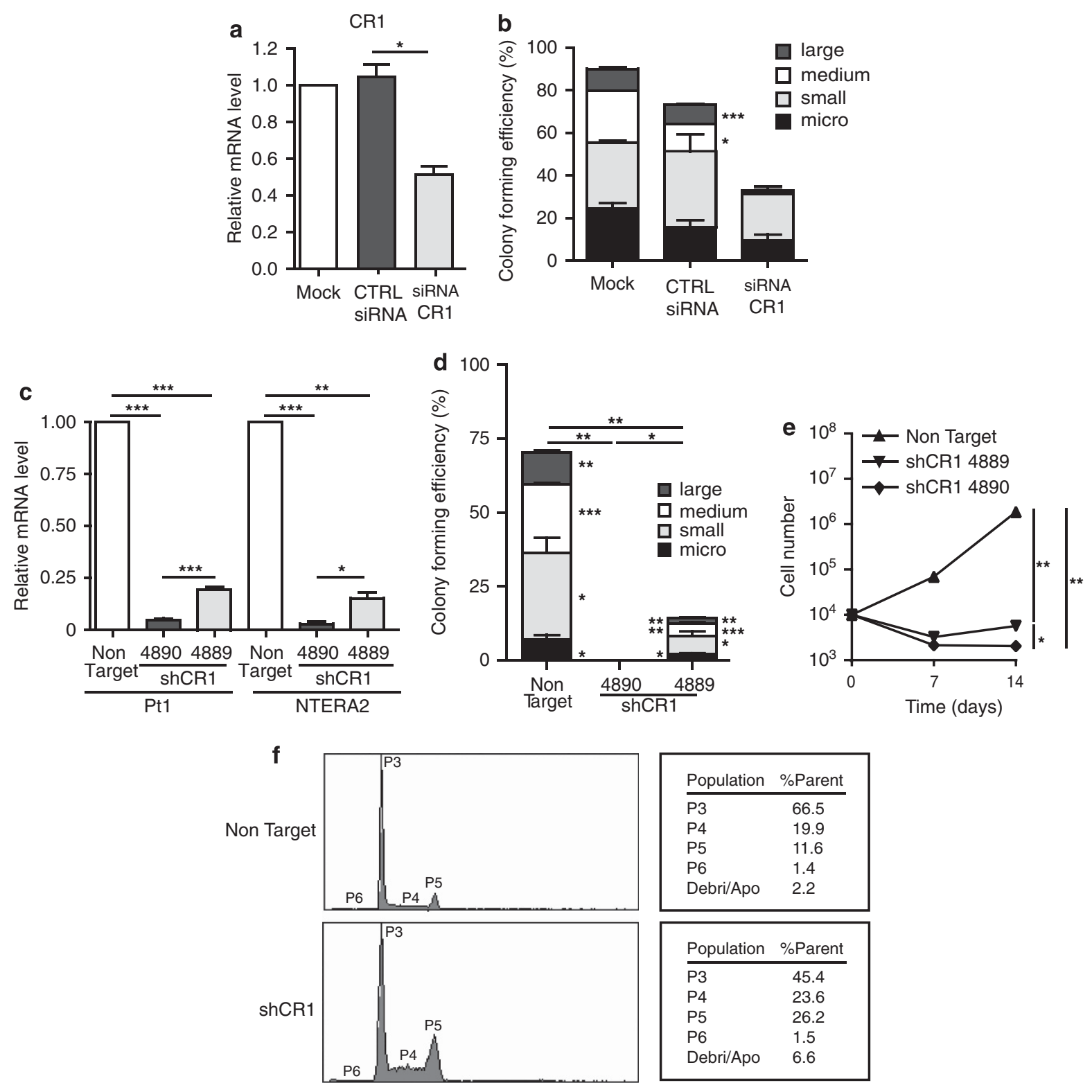

\begin{tabular}{|ll|}
\hline Population & \%Parent \\
\hline P3 & 45.4 \\
P4 & 23.6 \\
P5 & 26.2 \\
P6 & 1.5 \\
Debri/Apo & 6.6 \\
\hline
\end{tabular}
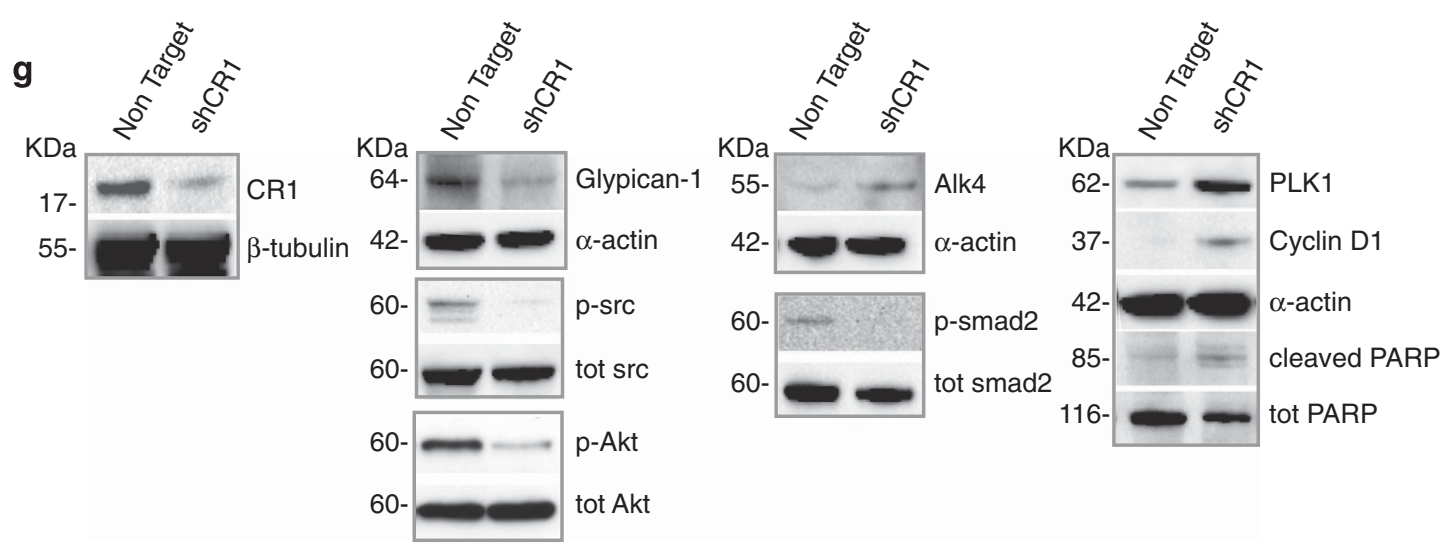
from Thermo Scientific/Life Technologies (Carlsbad, CA, USA). For stable CR1 silencing, the lentiviral pLKO.1 silencing vectors containing either a non-targeting sequence, the sh4889 or sh4890 sequences that target the $3^{\prime}$-UTR and the coding sequence of CR1, respectively, were purchased from Sigma-Aldrich. The sh4890 sequence was subsequently cloned in the TRIPZ lentiviral inducible vector (Thermo Scientific/ Life Technologies). The lentiviral reporter vector TWEEN-Luc-GFP was constructed by using pRRL-CMV-PGK-GFP-WPRE (pTWEEN) as a backbone for subcloning a firefly luciferase Nhel/Xbal cDNA fragment extracted from pGL3 (Promega) into the Xbal site at the $3^{\prime}$ of the CMV promoter.

Clonal marking. Six different lentiviral vectors were used to transduce spheroid cells: LeGO-C2 (mCherry), LeGO-V2 (Venus), LeGO-Cer2 (Cerulean), TWEEN-GFP (green), TWEEN-RedFP (red) and pLOX-CFP (Cyan). Cells were separated on the basis of fluorescent protein expression and single clones were derived from each fluorescent cell culture by single-cell automated sorting on a 96-well plate. After 1 month, three single-cell-derived spheres for each color were expanded, tested for fluorescent protein expression and one clone of each color was used for subsequent experiments.

Whole-mount in situ hybridization. A DNA template was generated from mouse cDNAs encoding CR1 (entire open reading frame). Antisense RNA probes were synthesized from $1 \mu \mathrm{g}$ of linearized cDNA and labeled with either digoxigeninUTP (Roche Diagnostics Corporation, Indianapolis, IN, USA) using T3, T7 and SP6 RNA polymerases (Promega). The transcription reaction was carried out at $37^{\circ} \mathrm{C}$ for $2 \mathrm{~h}$, followed by DNasel treatment (Promega). After the quality of the labeled RNA was assessed by gel electrophoresis, transcripts were ethanol precipitated in $7.5 \mathrm{M}$ $\mathrm{LiCl}$ and TE of pH 8.0 and stored overnight at $-80^{\circ} \mathrm{C}$. Probes were resuspended in hybridization solution and stored at $-80^{\circ} \mathrm{C}$. Mouse intestines were dissected, washed in PBS and fixed in $4 \%$ paraformaldehyde (PFA) in PBS at $4^{\circ} \mathrm{C}$. In situ hybridization was performed on $5 \mu \mathrm{m}$ paraffin-embedded sections. After rehydration in PBS containing $0.1 \%$ Tween20 (PBT), sections were treated with proteinase $K$ $(10 \mathrm{mg} / \mathrm{ml})$ for $3-20 \mathrm{~min}$, and then postixed in $4 \%$ PFA plus $0.2 \%$ glutaraldehyde. Proteinase $\mathrm{K}$ was inactivated with $0.2 \%$ glycine in PBT. All samples were incubated in hybridization buffer containing $50 \%$ formamide for $2 \mathrm{~h}$ at $70^{\circ} \mathrm{C}$ and then hybridized at the same temperature with $0.5 \mu \mathrm{g} / \mathrm{ml}$ labeled RNA probe overnight. Posthybridization washes and RNase A treatment $(100 \mu \mathrm{g} / \mathrm{ml})$ were subsequently performed. Tissues were rinsed $3 \times 5$ minutes in maleic acid buffer (MAB; $0.1 \mathrm{M}$ maleic acid of $\mathrm{pH} 7.5$, $0.15 \mathrm{M} \mathrm{NaCl}, 0.1 \%$ Tween20 and $0.002 \mathrm{M}$ levamisole) and then incubated in blocking buffer (MAB 10\% sheep serum, and $2 \%$ blocking reagent, Roche Diagnostics Corporation), at room temperature for $3 \mathrm{~h}$. Hybridized probes were incubated with preabsorbed anti-digoxigenin-AP antibody (Roche Diagnostics Corporation) diluted $1: 4000$ in blocking buffer (Roche Diagnostics Corporation) overnight at $4{ }^{\circ} \mathrm{C}$. Tissues were washed several times with $\mathrm{MAB}$ at room temperature, and then developed with BM purple (Roche Diagnostics Corporation) color substrate for 5-16 h. Reactions were then stopped with $3 \times 5$ minutes PBT washes, and samples postixed in $4 \%$ PFA plus $0.2 \%$ glutaraldehyde.

Immunofluorescence. Colon cancer spheroids were cytospun at low speed on polylysine-coated glass slides, whereas SDAC were grown on matrigel-coated coverslips. Typically, cells were fixed in $2 \%$ PFA and permeabilized in $0.1 \%$ Triton X-100 (Bio-Rad Laboratories, Hercules, CA, USA) then incubated overnight at $4{ }^{\circ} \mathrm{C}$ with primary antibodies (described in the Antibodies and Reagents section) dissolved in PBS containing 3\% BSA, 0,1\% Triton X-100. After two washes in PBS, cells were incubated with Alexa Fluor-conjugated secondary antibodies for $30 \mathrm{~min}$ at room temperature in the dark, stained for 15 min with DAPI (Invitrogen) diluited in
PBS 3\% BSA and subsequently mounted with Prolong-Gold antifade (Invitrogen). Immunofluorescence staining of FFPE normal and neoplastic human colon tissue sections was performed as follows: sections were deparaffinized, washed in PBS and incubated with anti-CR1 antibody ${ }^{40}\left(1: 50\right.$ in PBS) overnight at $4{ }^{\circ} \mathrm{C}$. After washing in PBS, sections were incubated with a FITC-conjugated secondary antibody (1:200 in PBS) $1 \mathrm{~h}$ at $37^{\circ} \mathrm{C}$ and then treated with $4 \%$ PFA for $30 \mathrm{~min}$ at room temperature. After an additional washing in PBS, sections were permeabilized with a brief incubation in a microwave oven with Epitope Retrieval $\mathrm{pH}-6$ (Novocastra-Leica Biosystems, Richmond, IL, USA) followed by 10 min incubation with cold $0.1 \%$ Triton X-100. After washing in PBS, sections were incubated overnight at $4{ }^{\circ} \mathrm{C}$ with anti-EphB2 (1:100 in PBS/3\% BSA/0.05 Tween20) or antiLgr5 (1:100 in PBS/3\% BSA/0.05 Tween20) antibodies. After incubation with rhodamine-conjugated secondary antibodies, nuclei were stained with TOTO-3 (Invitrogen Molecular Probes) and sections were mounted with Prolong-Gold antifade. Slides were analyzed on a FV1000 confocal microscope (Olympus, Tokyo, Japan) equipped with $40 \times$ and $60 \times$ oil immersion objectives and the Olympus Fluoview software.

Flow cytometry and sorting. Spheroid-dissociated cells were labeled with primary antibodies (PE-conjugated anti-Lgr5, Alexa Fluor 488 conjugated anti-EphB2 or non-conjugated anti-CR1, described in the Antibodies and Reagents section) for $1 \mathrm{~h}$ on ice and, where necessary, with secondary antibodies for $30 \mathrm{~min}$ on ice, then sorted with a FACSAria (BD Biosciences, San Jose, CA, USA) or analyzed with a FACSCanto flow cytometer equipped with a DIVA software (BD Biosciences). $10 \mu \mathrm{g} / \mathrm{ml}$ 7-aminoactinomycin D (Sigma-Aldrich) was always added for dead cell exclusion. The cell cycle status of colon CSC was assessed by staining dissociated spheroids with $50 \mu \mathrm{g} / \mathrm{ml}$ propidium iodide dissolved in buffer $0.1 \%$ trisodium citrate, $9.65 \mathrm{mM} \mathrm{NaCl}, 0.1 \% \mathrm{NP} 40,200 \mu \mathrm{g} / \mathrm{ml}$ RNAse for $1 \mathrm{~h}$ at room temperature. Analysis of debris/apoptotic cells was performed by gating the desired area of the plot on the basis of physical parameters and subsequent fluorescence analysis on a logarithmic scale.

Quantitative real-time PCR. Total RNA was extracted with TRlzol (Invitrogen) following manufacturer's instructions. $50 \mathrm{ng}$ of RNA were reverse transcribed with M-MLV reverse transcriptase (Invitrogen) and $200 \mathrm{ng}$ of cDNA were used as template in the PCR reactions. Specific primers (specified in Supplementary Table 3) were used for Nanog, CR1, Nodal, Glypican, ALK4 and GRP78, while a TaqMan assay (Applied Biosystems/Life Technologies, Carlsbad, CA, USA; Cat. 4351372) was used for Lgr5 amplification. Normalization was performed using $\beta$-actin as reference (TaqMan assay, Applied Biosystems/Life Technologies, Cat. 4310881E) for Nanog, CR1 and Lgr5 and GAPDH for Nodal, Glypican, ALK4 and GRP78. Values are expressed in terms of 2- $\Delta \Delta \mathrm{CT}$ where $\Delta \Delta \mathrm{CT}=\Delta \mathrm{CT}$ sample $-\Delta \mathrm{CT}$ calibrator. $\Delta \mathrm{CT}$ is the difference in threshold cycles between the specific RNA and $\beta$-actin amplicons, and CT is a parameter given by StepOne Plus Real-Time PCR software by negative correlation with an internal reference dye (ROX).

Western blotting. Cell lysates were obtained from approximately $2.5 \times 10^{5}$ spheroid cells by incubation of cell pellets in $1 \% \mathrm{NP} 40$ lysis buffer $(20 \mathrm{mM}$ Tris $\mathrm{HCl}$ pH $7.2,200 \mathrm{mM} \mathrm{NaCl}, 1 \% \mathrm{NP} 40$ ) supplemented with protease inhibitor cocktail and phosphatase inhibitor cocktails I and II (all from Sigma-Aldrich). Lysate concentrations were determined by the Bradford assay (Bio-Rad Laboratories) and equal amounts of proteins were loaded on a $12 \%$ precast gel (Invitrogen) and transferred to nitrocellulose membranes. Blots were blocked with TBST $5 \%$ nonfat dry milk and incubated overnight at $4{ }^{\circ} \mathrm{C}$ with primary antibodies (described in the Antibodies and Reagents section), then incubated for $45 \mathrm{~min}$ with secondary HRP-conjugated

Figure 5 CR1 silencing inhibits CSC growth and self renewal in vitro and reduces Src-Akt activation. (a) CR1 mRNA level in spheroid line Pt1 mock transfected (Mock), transfected with non-targeting small interfering RNA (CTRL siRNA) or with siRNA against CR1 (siRNA CR1), collected $24 \mathrm{~h}$ after transfection. ${ }^{*} P<0.05$ (b) Percentage of colonies generated by spheroid cells treated as in a. Asterisks refer to the statistical significance of large and medium colonies compared between CTRL siRNA and siRNA CR1 samples. ${ }^{\star} P<0.05$; ${ }^{* \star \star} P<0.001$. Differences in the total number of colonies between the three samples are non-significant (c) CR1 mRNA expression in the colon cancer spheroid line Pt1 and in the embryonic carcinoma cell line NTERA2 transduced with the pLKO vector containing either a non-targeting sequence (Non Target), the sh4889 or the sh4890 CR1-silencing sequences. ${ }^{*} P<0.05$; ${ }^{* \star *} P<0.001$ (d) Colony formation by spheroid cells of line Pt1 transduced as in $\mathbf{c}$. Asterisks flanking the histogram bars refer to the statistical significance of colony size sections belonging to different samples. From left to right: Non Target versus sh4890, sh4890 versus sh4889 and Non Target versus sh4889. ${ }^{\star} P<0.05 ;{ }^{* \star} P<0.01,{ }^{* \star *} P<0.001$ (e) Growth (logarithmic scale) of spheroid line Pt1 transduced as in $\mathbf{c},{ }^{*} P<0.05 ;{ }^{* \star} P<0.01$ (f) Cell cycle analysis of CSC transduced with the pLKO sh4890 CR1-silencing vector, 4 days after transduction. Right panels show the percentage of cells relative to each peak. Debri/apo refers to the percentage of events including hypodiploid cells and debris, calculated as described in the Materials and Methods section (g) Immunoblot analysis of the indicated proteins in control CSC (Non Target) and in CSC stably transduced with the pLKO sh4890 CR1-silencing vector (shCR1). PLK1, polo-like kinase 1; PARP, poly ADP ribose polymerase 
antibodies dissolved in TBST 1\% BSA. Chemiluminescent signals were detected with Super Signal West Pico (Pierce Pierce, Waltham, MA, USA). For subcellular fractionation, the $Q$ Proteome Cell Compartment Kit was used following the manufacturer's instructions (Qiagen, Germantown, MD, USA).
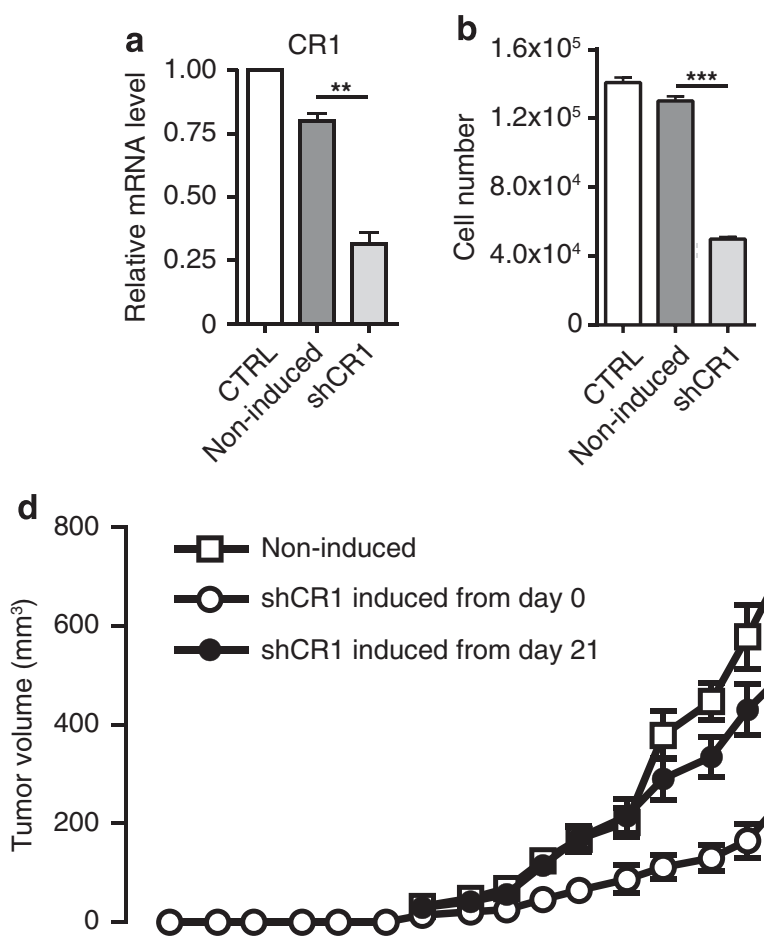$$
0
$$

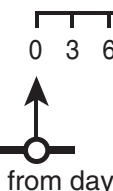

Doxy from day 0

Doxy from day 21
ELISA assay. 96-well plates were coated with $4 \mu \mathrm{g} / \mathrm{ml}$ of mouse anti-human CR1 Ab (R\&D Systems Inc., Cat. 842077) in PBS (137 mM NaCl; 2,7 mM KCl; 8,1

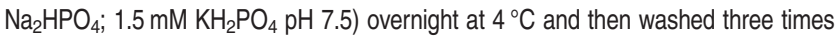
with PBS-Tween20 0.05\%. Aspecific binding sites were blocked with PBS-BSA $1 \%$

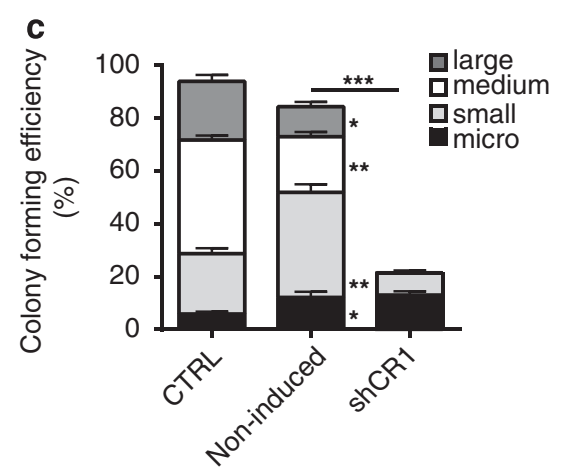

e

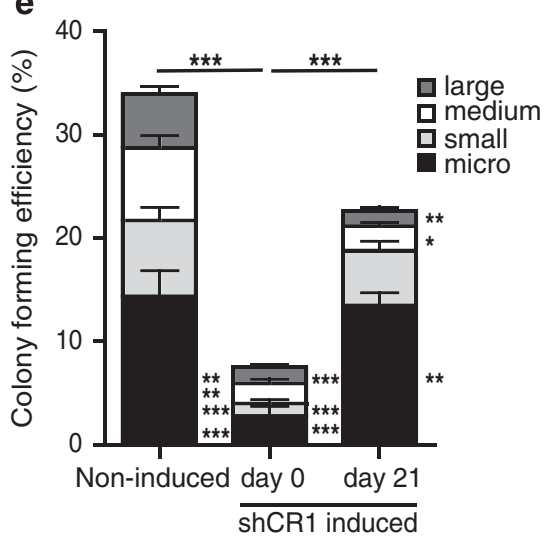

f

\begin{tabular}{|c|c|c|c|c|c|c|}
\hline \multicolumn{2}{|c|}{ TUMOR RATIO } & \multicolumn{2}{|c|}{$\begin{array}{l}\text { TUMOR ONSET } \\
\text { (WEEKS FROM } \\
\text { INJECTION) }\end{array}$} & \multicolumn{2}{|c|}{$\begin{array}{l}\text { TUMOR MEDIAN SIZE } \\
\text { mm³ }^{3}(16 \text { WEEKS) }\end{array}$} & \\
\hline $4 / 4$ & $3 / 4$ & 4 & 7 & $1479.625 \pm 987.55$ & $851.6 \pm 300$ & 100 CELLS \\
\hline $3 / 4$ & $2 / 4$ & 6 & 10 & $1567.45 \pm 510$ & $800.8 \pm 250.1$ & 30 CELLS \\
\hline $2 / 4$ & $0 / 4$ & 8 & - & $696.95 \pm 59.95$ & - & 10 CELLS \\
\hline Non-induced & shCR1 & Non-induced & shCR1 & Non-induced & shCR1 & \\
\hline
\end{tabular}

Figure 6 Cripto silencing inhibits tumor progression and decreases CSC numbers and tumor forming capacity in vivo. (a) mRNA levels, measured $48 \mathrm{~h}$ after infection, of spheroid line Pt1 non-transduced (CTRL) or transduced with the inducible CR1 silencing lentiviral vector TRIPZ sh4890 in the absence or in the presence of doxycycline (Noninduced and shCR1, respectively). ${ }^{* *} P<0.01$. The difference between CTRL and Non-induced samples is non-significant (b) Cell numbers of Pt1 spheroid line treated as in a. ${ }^{* \star *} P<0.001$ (c) Size and normalized number of colonies formed in soft agar by spheroid line Pt1 treated as in $\mathbf{a} .{ }^{*} P<0.05$; ${ }^{* *} P<0.01,{ }^{* * *} P<0.001$. Asterisks on the side of bars refer to the statistical significance of colony size sections compared between Non-induced and shCR1 samples (d) In vivo growth of tumor xenografts obtained by subcutaneous inoculation of Pt1 spheroids transduced with TRIPZ sh4890, non-induced (Non-induced) or in which CR1 silencing was induced by treating mice with doxycycline from day 0 (shCR1 induced from day 0 ) or at the time of tumor appearance (shCR1 induced from day 21). Results shown are the mean \pm S.D. of values obtained using six mice per group. ${ }^{*} P<0.05$; ${ }^{* *} P<0.01,{ }^{* * *} P<0.001$ (e) Size and normalized number of colonies formed in soft agar by xenografts cells at the end of the experiment shown in d. ${ }^{*} P<0.05$; ${ }^{* *} P<0.01,{ }^{* *} P<0.001$. Asterisks on the side of bars refer to the statistical significance of colony size sections belonging to different samples. From left to right: Vector versus day 0 , day 0 versus day 21 , day 21 versus Vector. ${ }^{*} P<0.05$; ${ }^{\star \star} P<0.01,{ }^{\star \star \star} P<0.001$ (f) Results of limiting dilution serial transplants performed with cells extracted from subcutaneous xenografts expressing the TRIPZ sh4890 CR1-silencing sequence without doxycycline induction (Non-induced) or induced with doxycycline at the time of injection (shCR1) shown in $\mathbf{d}$. Cells were taken from dissociated xenografts at the end of the experiment, kept in the culture for one night in the presence or in the absence of doxycycline and subcutaneously inoculated at the indicated numbers (100,30 and 10 cells) in secondary recipients (four mice for each condition). Doxycycline was added to the drinking water of secondary mice of the shCR1 group as described in Materials and Methods 
a

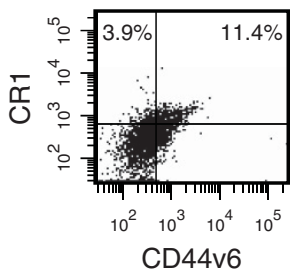

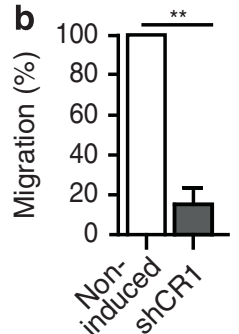

C

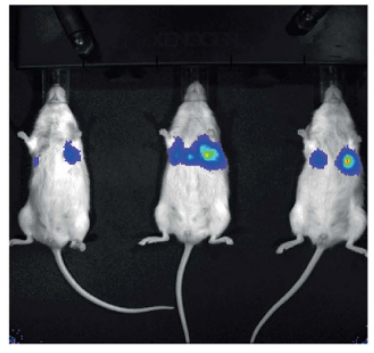

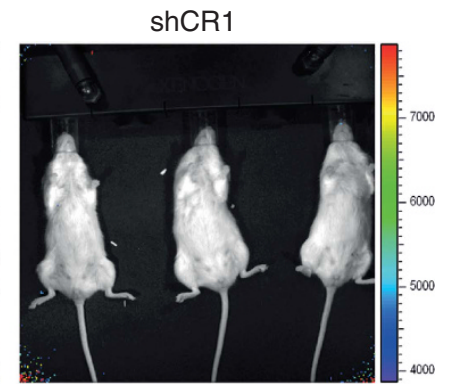

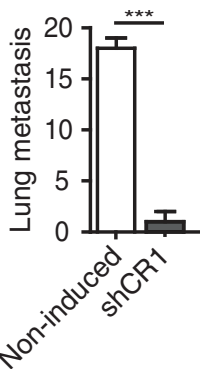

e Non-induced
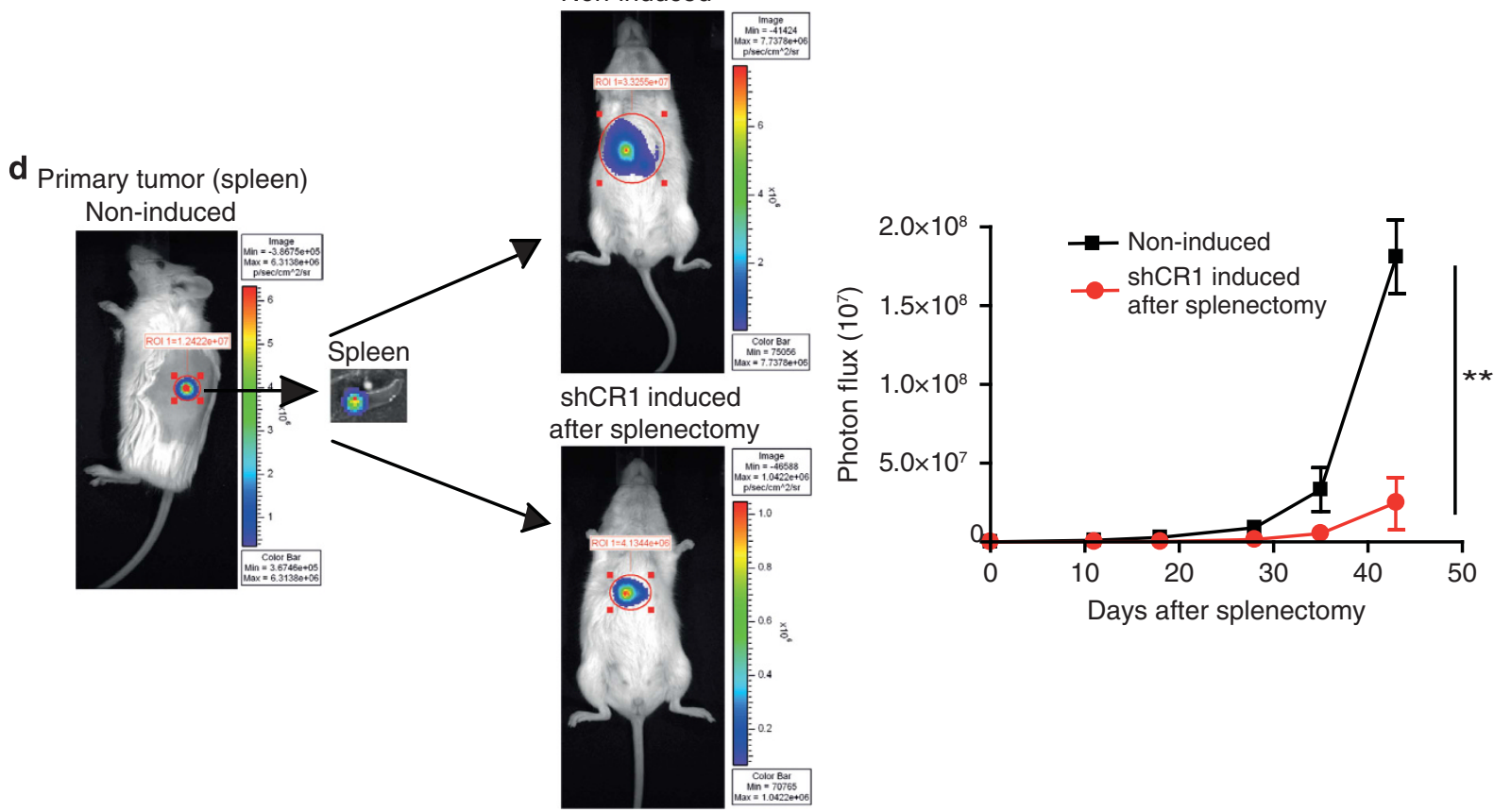

Figure 7 CR1 is essential for CSC migration and growth at metastatic sites. (a) Flow cytometry analysis of CR1 and CD44v6 in colon CSC line Pt1 (b) Percentage of migrated CSC in samples transduced with the TRIPZ sh4890 vector in the absence of doxycycline (Non-induced) or in the presence of doxycycline, which activates CR1 silencing mediated by the sh4890 RNA (shCR1) (c) Formation of lung tumors by colon cancer spheroids (line Pt1) transduced with TRIPZ sh4890 in the absence or in the presence of doxycycline (Non-induced and shCR1, respectively), detected 4 months following caudal vein injection. Left: representative picture of mice 4 months after injection; right: quantification of lung tumors derived from three different experiments. ${ }^{* *} P<0.001$ (d) Mice (eight) were inoculated in the spleen with CSCs transduced as above and left without doxycycline (Noninduced) to allow the growth of splenic xenografts (left panel). After 1 month, the spleen was removed and mice were divided into two groups, one of which was left untreated (Noninduced) while the other received doxycycline in the drinking water to activate CR1 silencing (shCR1 after splenectomy). Mice were monitored for the growth of liver metastases (central panels), which were quantified by bioimaging (right graph). ${ }^{\star \star} P<0.01$

(300 $\mu$ l/well) for at least $1 \mathrm{~h}$ at RT. After washing, the concentrated cell media $(1: 3$ of starting volume) were added and incubated overnight at $4{ }^{\circ} \mathrm{C}$. The plates were incubated with $62.5 \mathrm{ng} / \mathrm{ml}$ of biotinylated anti-human CR1 Ab (R\&D Systems Inc., Cat. 842079 ) in Reagent Diluent (1\% BSA in PBS $1 \times$ pH 7.5) for $2 \mathrm{~h}$ at RT. Finally, the plates were incubated for 20 min at RT with streptavidin complex conjugated with horseradish peroxidase (R\&D Systems Inc.). The signals were then visualized with o-phenylenediamineperoxidase substrate (Sigma-Aldrich) and the relative absorbance was read at $490 \mathrm{~nm}$ on a Benchmark microplate reader (Bio-Rad Laboratories).

Immunohistochemistry. Intestinal tissues were fixed in 4\% PFA in PBS overnight at $4{ }^{\circ} \mathrm{C}$ and were paraffin embedded. For immunohistochemistry, tissues cut in $5-\mu \mathrm{m}$ sections were deparaffinized in xylene and rehydrated in a series of 
graded ethanol washes. Endogenous peroxidase activity was blocked with $3 \% \mathrm{H}_{2} \mathrm{O}_{2}$ in methanol for $10 \mathrm{~min}$. Antigen retrieval was performed by autoclaving slides in citrate buffer (pH 6.0). CR1 protein was stained with anti-mouse-CR1 mAb (B3F6, Biogen Idec, Weston, MA, USA) and the Vectastain ABC kit (Vector Laboratories Inc, Burlingame, CA, USA) following the manufacturer's instructions. Sections were counterstained with hematoxylin.

Motility assay. $1 \times 10^{4}$ control or CR1-interfered CSCs were suspended in $200 \mu \mathrm{l}$ of non-supplemented stem cell medium and plated into the upper wells of matrigel-coated modified Boyden Chambers containing porous $8 \mathrm{~mm}$ diameter polycarbonate membranes (Costar Scientific Corporation, Cambridge, MA, USA). Lower wells contained $500 \mu \mathrm{l}$ of stem cell medium supplemented with $20 \mathrm{ng} / \mathrm{ml}$ EGF and $10 \mathrm{ng} / \mathrm{ml}$ basic FGF. After 4 days, the cells in the upper wells were removed, whereas the cells that migrated to the lower wells were fixed, stained with DAPI in PBS 1\% NP40 for $5 \mathrm{~min}$ and counted under a fluorescence microscope. The amount of migrated cells was quantified with the ImageJ program.

In vivo experiments. All animal experiments were conducted according to the national Animal Experimentation guidelines (D.L.116/92) upon approval by the ISS Animal Experimentation Committee. For tumorigenesis assays, female 6-8 weeks old NSG mice were subcutaneously injected with $10^{3}$ dissociated spheroid cells resuspended in $100 \mu$ l Matrigel - CSC medium 1:1. Tumor volume was evaluated by using an external digital caliper. For in vivo inducible CR1 silencing, doxycycline $200 \mu \mathrm{g} / \mathrm{ml}$ was kept in the drinking water throughout the experiment. For experiments of CSC homing/metastasis, $10^{4}$ dissociated spheroid cells were injected into the caudal vein and tumor formation was monitored with an IVIS imaging system (Perkin Elmer, Waltham, MA, USA). For serial transplantation experiments, cells derived from dissociated xenografts were kept in culture overnight in the presence or in the absence of doxycycline and inoculated subcutaneously. Doxycycline was provided to mice throughout the experiment. Quantification of stem cell numbers was performed with the ELDA method. ${ }^{25}$ For heterotopic spleen injection NSG mice were anesthetized with a mixture of ketamine $(100 \mathrm{mg} / \mathrm{kg})$ and xylazine $(10 \mathrm{mg} / \mathrm{kg})$ injected into the peritoneal cavity. $2 \times 10^{4}$ CSC transduced with TRIPZ sh4890 and TWEEN-LUC-GFP were injected in the spleen during open laparotomy and mice were monitored with IVIS. After primary tumor formation the spleen was removed and doxycycline was added in the drinking water of the shCR1 group. Liver metastasis growth was monitored with IVIS once a week for six weeks.

Statistical analysis. Data were analyzed using the $\mathrm{R}$ software with non-paired double-tailed $t$ test after verifying normal distribution of the population with ShapiroWilk test. Results are presented as the mean \pm S.D. Statistical significance is expressed as ${ }^{\star} P$-value $0.01-0.05 ;{ }^{* \star} P$-value $0.001-0.01$ and ${ }^{* \star *} P$-value $<0.001$.

\section{Conflict of Interest}

The authors declare no conflict of interest.

Acknowledgements. We thank Stefano Guida and Gennaro Andolfi for excellent technical assistance, Alessandro Giuliani for helpful discussion, Boris Fehse and Kristoffer Riecken for providing the LeGO vectors, Anna Rizzo for help with statistical analysis, Valeria Coppola for help with the Oncomine database and Mario Falchi for image quantification. This work was supported by a Ricerca Finalizzata grant (Italian Ministry of Health) and AIRC IG grant 15749 to AZ, AIRC 5 per Mille 9979 grant to RDM and GS, AIRC IG grant 11599, Epigenomics Flagship Project (EPIGEN) MIUR-CNR and Italian Ministry of Education-University-Research (MIURPRIN) grants to GM.

\section{Author contributions}

FF performed the majority of experiments and essentially contributed to the experimental design, PC, GG, AF, NPC, CP, MT and KW performed experiments, MLDA and MB performed in vivo tumorigenicity and limiting dilution assays, AP provided the reporter constructs for metastasis assays, $A B$ performed FACS analyses, DS, GM, GS and RDM provided essential expertize on CR1 and CSC, respectively. $A Z$ designed and supervised the work, performed experiments and wrote the paper.

1. Sanchez Alvarado A, Yamanaka S. Rethinking differentiation: stem cells, regeneration, and plasticity. Cell 2014; 157: 110-119.

2. Kreso A, Dick JE. Evolution of the cancer stem cell model. Cell Stem Cell 2014; 14: 275-291.
3. Macfarlan TS, Gifford WD, Driscoll S, Lettieri K, Rowe HM, Bonanomi D et al. Embryonic stem cell potency fluctuates with endogenous retrovirus activity. Nature 2012; 487: $57-63$.

4. van Es JH, Sato T, van de Wetering M, Lyubimova A, Nee ANY, Gregorieff A et al. Dll1+ secretory progenitor cells revert to stem cells upon crypt damage. Nat Cell Biol 2012; 14: 1099-1104.

5. Schwitalla S, Fingerle AA, Cammareri P, Nebelsiek T, Goktuna SI, Ziegler PK et al. Intestinal tumorigenesis initiated by dedifferentiation and acquisition of stem-cell-like properties. Cell 2013; 152: 25-38.

6. Chaffer CL, Brueckmann I, Scheel C, Kaestli AJ, Wiggins PA, Rodrigues LO et al. Normal and neoplastic nonstem cells can spontaneously convert to a stem-like state. Proc Natl Acad Sci USA 2011; 108: 7950-7955.

7. Gupta PB, Fillmore CM, Jiang G, Shapira SD, Tao K, Kuperwasser C et al. Stochastic state transitions give rise to phenotypic equilibrium in populations of cancer cells. Cell 2011; 146: 633-644.

8. Vermeulen L, Snippert HJ. Stem cell dynamics in homeostasis and cancer of the intestine. Nat Rev Cancer 2014; 14: 468-480.

9. Zeuner A, Todaro M, Stassi G, De Maria R. Colorectal cancer stem cells: from the crypt to the clinic. Cell Stem Cell 2014; 15: 692-705.

10. Bianco C, Rangel MC, Castro NP, Nagaoka T, Rollman K, Gonzales M et al. Role of Cripto-1 in stem cell maintenance and malignant progression. Am J Pathol 2010; 177: 532-540.

11. Guardiola $\mathrm{O}$, Lafuste $\mathrm{P}$, Brunelli $\mathrm{S}$, laconis $\mathrm{S}$, Touvier T, Mourikis $\mathrm{P}$ et al. Cripto regulates skeletal muscle regeneration and modulates satellite cell determination by antagonizing myostatin. Proc Natl Acad Sci USA 2012; 109: E3231-E3240.

12. Adamson ED, Minchiotti G, Salomon DS. Cripto: a tumor growth factor and more. J Cell Physiol 2002; 190: 267-278.

13. Wechselberger C, Strizzi L, Kenney N, Hirota M, Sun Y, Ebert A et al. Human Cripto-1 overexpression in the mouse mammary gland results in the development of hyperplasia and adenocarcinoma. Oncogene 2005; 24: 4094-4105.

14. Hough SR, Laslett AL, Grimmond SB, Kolle G, Pera MF. A continuum of cell states spans pluripotency and lineage commitment in human embryonic stem cells. PLoS One 2009; 4: e7708.

15. Watanabe K, Meyer MJ, Strizzi L, Lee JM, Gonzales M, Bianco C et al. Cripto-1 is a cell surface marker for a tumorigenic, undifferentiated subpopulation in human embryonal carcinoma cells. Stem Cells 2010; 28: 1303-1314.

16. Ricci-Vitiani L, Lombardi DG, Pilozzi E, Biffoni M, Todaro M, Peschle $C$ et al. Identification and expansion of human colon-cancer-initiating cells. Nature 2007; 445: 111-115.

17. Vermeulen L, Todaro M, de Sousa Mello F, Sprick MR, Kemper K, Perez Alea M et al. Singlecell cloning of colon cancer stem cells reveals a multi-lineage differentiation capacity. Proc Natl Acad Sci USA 2008; 105: 13427-13432.

18. Dieter SM, Ball CR, Hoffmann CM, Nowrouzi A, Herbst F, Zavidij O et al. Distinct types of tumor-initiating cells form human colon cancer tumors and metastases. Cell Stem Cell 2011; 9: 357-365.

19. Snippert HJ, van Es JH, van den Born M, Begthel H, Stange DE, Barker N et al. Prominin-1/ CD133 marks stem cells and early progenitors in mouse small intestine. Gastroenterology 2009; 136: 2187-2194 e2181.

20. Weber K, Thomaschewski M, Warlich M, Volz T, Cornils K, Niebuhr B et al. RGB marking facilitates multicolor clonal cell tracking. Nat Med 2011; 17: 504-509.

21. Minchiotti G, Parisi S, Liguori G, Signore M, Lania G, Adamson ED et al. Membraneanchorage of Cripto protein by glycosylphosphatidylinositol and its distribution during early mouse development. Mech Dev 2000; 90: 133-142.

22. Watanabe K, Hamada S, Bianco C, Mancino M, Nagaoka T, Gonzales M et al. Requirement of glycosylphosphatidylinositol anchor of Cripto-1 for trans activity as a Nodal co-receptor. J Biol Chem 2007; 282: 35772-35786.

23. Strizzi L, Bianco C, Normanno N, Salomon D. Cripto-1: a multifunctional modulator during embryogenesis and oncogenesis. Oncogene 2005; 24: 5731-5741.

24. Bianco C, Mysliwiec M, Watanabe K, Mancino M, Nagaoka T, Gonzales M et al. Activation of a Nodal-independent signaling pathway by Cripto-1 mutants with impaired activation of a Nodal-dependent signaling pathway. FEBS Lett 2008; 582: 3997-4002.

25. Hu Y, Smyth GK. ELDA: extreme limiting dilution analysis for comparing depleted and enriched populations in stem cell and other assays. J Immunol Methods 2009; 347: 70-78.

26. Todaro M, Gaggianesi M, Catalano V, Benfante A, lovino F, Biffoni M et al. CD44v6 is a marker of constitutive and reprogrammed cancer stem cells driving colon cancer metastasis. Cell Stem Cell 2014; 14: 342-356.

27. MacArthur BD, Sevilla A, Lenz M, Muller FJ, Schuldt BM, Schuppert AA et al. Nanog-dependent feedback loops regulate murine embryonic stem cell heterogeneity. Nat Cell Biol 2012; 14: 1139-1147.

28. Kalmar T, Lim C, Hayward P, Munoz-Descalzo S, Nichols J, Garcia-Ojalvo J et al. Regulated fluctuations in nanog expression mediate cell fate decisions in embryonic stem cells. PLoS Biol 2009; 7: e1000149.

29. Hayashi K, Lopes SM, Tang F, Surani MA. Dynamic equilibrium and heterogeneity of mouse pluripotent stem cells with distinct functional and epigenetic states. Cell Stem Cell 2008; 3: $391-401$. 
30. Abranches E, Guedes AM, Moravec M, Maamar H, Svoboda P, Raj A et al. Stochastic NANOG fluctuations allow mouse embryonic stem cells to explore pluripotency Development 2014; 141: 2770-2779.

31. MacArthur BD, Lemischka IR. Statistical mechanics of pluripotency. Cell 2013; 154 484-489.

32. MacArthur BD. Collective dynamics of stem cell populations. Proc Natl Acad Sci USA 2014 111: $3653-3654$

33. Vainstein V, Kirnasovsky OU, Kogan Y, Agur Z. Strategies for cancer stem cell elimination: insights from mathematical modeling. J Theor Biol 2012; 298: 32-41.

34. Tsuchiya M, Wong ST, Yeo ZX, Colosimo A, Palumbo MC, Farina L et al. Gene expression waves. Cell cycle independent collective dynamics in cultured cells. FEBS J 2007; 274 2878-2886.

35. Ding J, Yang L, Yan YT, Chen A, Desai N, Wynshaw-Boris A et al. Cripto is required for correct orientation of the anterior-posterior axis in the mouse embryo. Nature 1998; 395 : 702-707.

36. Ciardiello F, Kim N, Saeki T, Dono R, Persico MG, Plowman GD et al. Differential expression of epidermal growth factor-related proteins in human colorectal tumors. Proc Natl Acad Sci USA 1991; 88: 7792-7796.

37. Miyoshi N, Ishii H, Mimori K, Sekimoto M, Doki Y, Mori M. TDGF1 is a novel predictive marker for metachronous metastasis of colorectal cancer. Int J Oncol 2010; 36: 563-568.
38. Merlos-Suarez A, Barriga FM, Jung P, Iglesias M, Cespedes MV, Rossell $D$ et al. The intestinal stem cell signature identifies colorectal cancer stem cells and predicts disease relapse. Cell Stem Cell 2011; 8: 511-524.

39. Francescangeli F, Patrizii M, Signore M, Federici G, Di Franco S, Pagliuca A et al. Proliferation state and polo-like kinase 1 dependence of tumorigenic colon cancer cells. Stem Cells 2012; 30: 1819-1830.

40. Minchiotti G, Manco G, Parisi S, Lago CT, Rosa F, Persico MG. Structure-function analysis of the EGF-CFC family member Cripto identifies residues essential for nodal signalling. Development 2001; 128: 4501-4510.

(c) (i) $(ङ)$ This work is licensed under a Creative Commons Attribution-NonCommercial-NoDerivs 4.0 International License. The images or other third party material in this article are included in the article's Creative Commons license, unless indicated otherwise in the credit line; if the material is not included under the Creative Commons license, users will need to obtain permission from the license holder to reproduce the material. To view a copy of this license, visit http://creativecommons.org/licenses/by-nc-nd/4.0/

Supplementary Information accompanies this paper on Cell Death and Differentiation website (http://www.nature.com/cdd) 